\title{
Contrasting Behaviors of FA and MA Cations in $\mathrm{APbBr}_{3}$
}

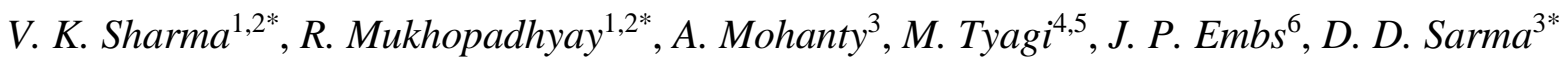

${ }^{1}$ Solid State Physics Division, Bhabha Atomic Research Centre, Mumbai 400085, India

${ }^{2}$ Homi Bhabha National Institute, Anushaktinagar, Mumbai 400094, India

${ }^{3}$ Solid State and Structural Chemistry Unit, Indian Institute of Science, Bengaluru 560012,

India

${ }^{4}$ National Institute of Standards and Technology, Center for Neutron Research, Gaithersburg, MD 20899, United States

${ }^{5}$ Department of Materials Science and Engineering, University of Maryland, College Park, MD 20742, United States

${ }^{6}$ Laboratory for Neutron Scattering and Imaging, Paul Scherrer Institut, 5232 Villigen PSI, Switzerland

*Email: sharmavk@barc.gov.in (VKS); ramaprosad@gmail.com (RM); sarma@iisc.ac.in (DDS) 


\section{Experimental Methods}

Sample preparations: Methylammonium lead bromide $\left(\mathrm{MAPbBr}_{3}\right)$ was synthesized from 1 Molar solution of $\mathrm{MABr}$ and $\mathrm{PbBr}_{2}$ (1:1 stoichiometry) in $\mathrm{N}, \mathrm{N}$-dimethylformamide. This solution was stirred at room temperature for $40 \mathrm{~min}$ and then drop-cast on preheated-glass slides (at $140{ }^{\circ} \mathrm{C}$ ). The orange powder and small crystals of $\mathrm{MAPbBr}_{3}$ were obtained upon solvent evaporation, powdered and stored in the glove box. For synthesising formamidinium lead bromide $\left(\mathrm{FAPbBr}_{3}\right)$, equimolar quantity of $\mathrm{FABr}$ and $\mathrm{PbBr}_{2}$ were dissolved in $\mathrm{N}, \mathrm{N}$ dimethylformamide, stirred for $1 \mathrm{~h}$, to get $1 \mathrm{M}$ solution. This solution was drop-cast on preheated-glass slides $\left(\right.$ at $170{ }^{\circ} \mathrm{C}$ ) inside a glovebox. After $20 \mathrm{~min}$ of heating, the orange powder and small crystals were obtained, powdered, and stored in the glove box.

Neutron Elastic Intensity Scan Measurements: Neutron elastic intensity scan, or elastic fixed window scan (EFWS), is a very suitable technique for investigating the phase transitions associated with temperature-activated microscopic mobility. In this scan, temperature of the sample is varied with small steps and at each temperature; the elastic intensity is measured for a constant time. Elastic intensity scan measurements have been carried out on $\mathrm{MAPbBr}_{3}$ and $\mathrm{FAPbBr}_{3}$ using High Flux Backscattering Spectrometer $(\mathrm{HFBS})^{1}$ at the NIST Center for Neutron Research (NCNR), Maryland. HFBS spectrometer has an energy resolution of $0.8 \mu \mathrm{eV}$ (Full Width at Half Maximum (FWHM)), which is particularly suitable for an elastic intensity scan measurement. Standard configuration with Si (111) as monochromator and analyzer crystals corresponding to incident neutron wavelength of $6.27 \AA$ was used. Measurements were carried out in the temperature range $4 \mathrm{~K}-295 \mathrm{~K}$ with a ramp rate of $0.8 \mathrm{~K} / \mathrm{min}$. At each temperature, count time was set to $1 \mathrm{~min}$.

Quasielastic Neutron Scattering (QENS) Experiments: While elastic intensity scan was used to study phase transitions associated with the cation dynamics, QENS technique is employed to investigate the dynamical behavior of a species in nanoseconds to picoseconds 
time scale. It provides information on the time scale, geometry of the motion and the potential that the species experiences. QENS experiments were carried out on $\mathrm{MAPbBr}_{3}$ and $\mathrm{FAPbBr}_{3}$ using the HFBS $(\Delta \mathrm{E}=0.8 \mu \mathrm{eV})$ spectrometer $^{1}$ at NCNR, USA, and the hybrid time of flight, FOCUS spectrometer ${ }^{2}$ at SINQ, Paul Scherrer Institute, Switzerland. FOCUS spectrometer was used with an incident neutron wavelength of $6 \AA$ providing an energy resolution (FWHM) of $\Delta \mathrm{E} \sim 45 \mu \mathrm{eV}$. QENS experiments were carried out on both the systems in the temperature range of 50-350 K. The samples were placed in annular aluminium sample holders with $0.2 \mathrm{~mm}$ internal spacing such that the scattering from the samples was no more than $10 \%$, thereby minimizing multiple scattering effects. Instrument resolution was obtained by measuring the spectra on standard vanadium samples. We have employed DAVE software ${ }^{3}$ to carry out standard data reduction including background subtraction, detector efficiency corrections, etc.

\section{Description of the QENS Data}

To understand the cation dynamics in $\mathrm{MAPbBr}_{3}$ and $\mathrm{FAPbBr}_{3}$, QENS measurements were carried out in the temperature ranges of $50-350 \mathrm{~K}$ using two different spectrometers with different energy windows namely, $\operatorname{HFBS}^{1}(\Delta \mathrm{E}=0.8 \mu \mathrm{eV})$ and $\operatorname{FOCUS}^{2}(\Delta \mathrm{E}=45 \mu \mathrm{eV})$, thus
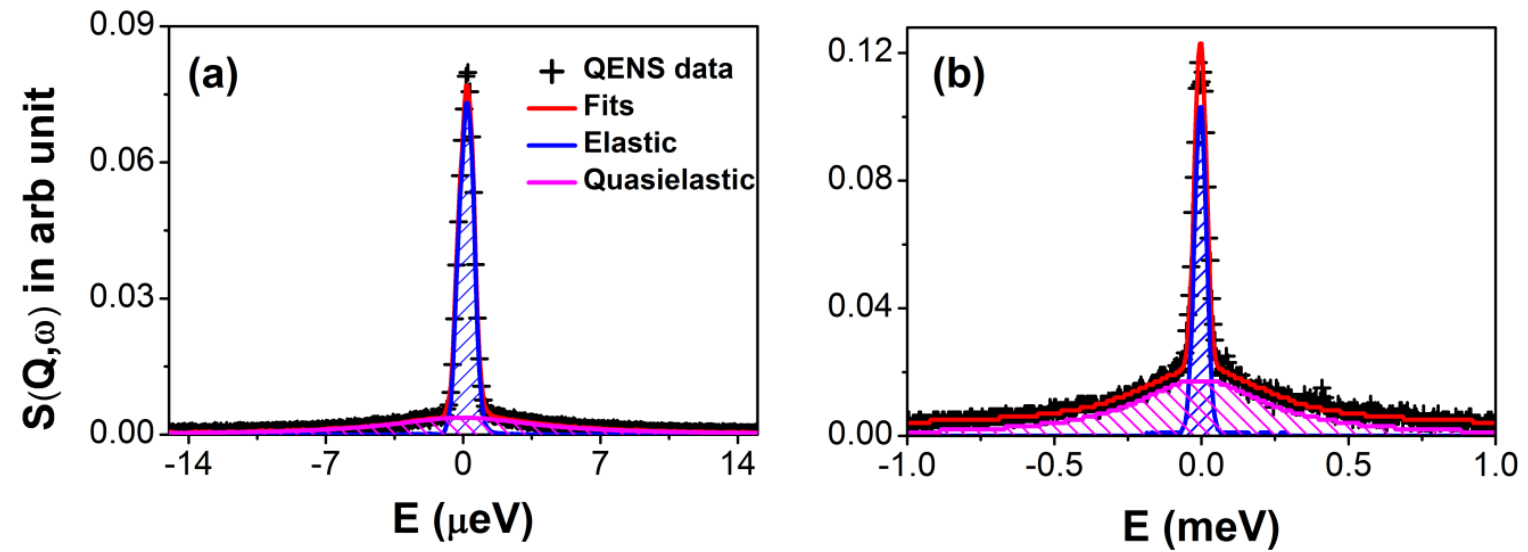

Figure S1. Typical fitted QENS spectra for (a) MAPbBr3 at 50K using HFBS spectrometer and (b) FAPbBr 3 at $300 \mathrm{~K}$ using FOCUS spectrometer, at $Q=1.4 \AA^{-1}$ assuming the model scattering function given in Eq. (1) of the MS. 
providing a wide dynamic range. The measured QENS spectra from these systems could be described by the scattering function containing a sum of an elastic and a quasielastic components (Eq. 1 in the MS). Typical fitted QENS spectra for $\mathrm{MAPbBr}_{3}$ and $\mathrm{FAPbBr}_{3}$ are shown in Figure $\mathrm{S} 1$

\section{Description of reorientational motion of MA/FA Cation using fractional isotropic rotational diffusion model}

In fractional isotropic rotational model, a fraction of cations is assumed to undergo isotropic rotation on a spherical surface. Elastic incoherent structure factor (EISF), $A(Q)$ for this model can be written as ${ }^{4}$

$$
A(Q)=\left[p_{x}+\left(1-p_{x}\right)\left(j_{0}(Q R)\right)^{2}\right]
$$

where $\left(1-p_{x}\right)$ is the fraction of mobile cation, $R$ is the radius of the rotation and $j_{0}$ is the spherical Bessel function of the zeroth order. The radius of rotation is actually the average distance of the hydrogen atoms from the centre of mass, which is 1.8 and $1.48 \AA$ for $\mathrm{FAPbBr}_{3}$ and $\mathrm{MAPbBr}_{3}$, respectively. The fraction of immobile cations $\left(p_{x}\right)$ is obtained by least square fittings of the experimental EISF. As shown in Figure $5 b$ of the manuscript this model describes the data for $\mathrm{FAPbBr}_{3}$ very well at all temperatures. For direct comparison between the parameters obtained from $\mathrm{MAPbBr}_{3}$ and $\mathrm{FAPbBr}_{3}$, the fractional isotropic rotational diffusion model has also been used to describe the EISF for $\mathrm{MAPbBr}_{3}$ and it is found to be quite satisfactory as shown in Figure S2. The obtained fractions of mobile cations for both the systems are shown in Figure 5c. 


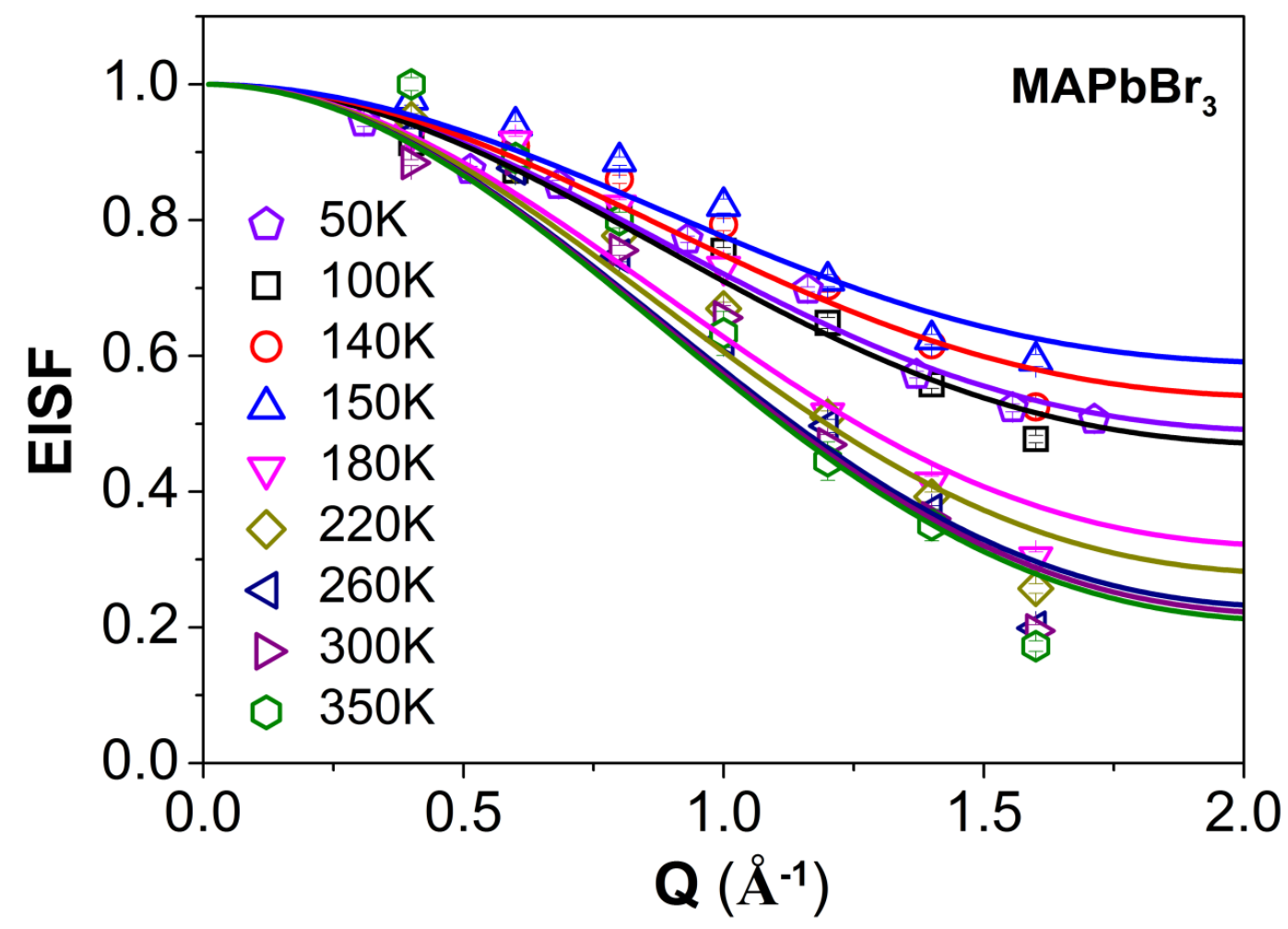

Figure S2. EISF for MAPbBr3 as described assuming fractional isotropic rotational model at different temperatures.

\section{Estimation of Time Scale of Reorientational Motion of MA and FA Cations}

Dynamic structure factor for fractional isotropic rotational model can be written as ${ }^{4}$

$$
S(Q, \omega)=p_{x} \delta(\omega)+\left(1-p_{x}\right)\left[j_{0}^{2}(Q r) \delta(\omega)+\frac{1}{\pi} \sum_{l=1}^{\infty}(2 l+1) j_{l}^{2}(Q r) \frac{l(l+1) D_{r}}{\left(l(l+1) D_{r}\right)^{2}+\omega^{2}}\right]
$$

where $j_{l}$ is the spherical Bessel functions of the $l^{\text {th }}$ order and $D_{r}$ is the rotational diffusion coefficient. Eq. (S1) is used to describe the behavior of half width at half maximum $(\Gamma)$ of the Lorentzian function. The reorientational rate for the cations in $\mathrm{MAPbBr}_{3}$ and $\mathrm{FAPbBr}_{3}$ are obtained from inverse of $D_{r}$. The behavior of $\Gamma$ with $Q$ at $300 \mathrm{~K}$ for both the systems, within the fractional isotropic rotational diffusion model, are shown in Figure S3. 


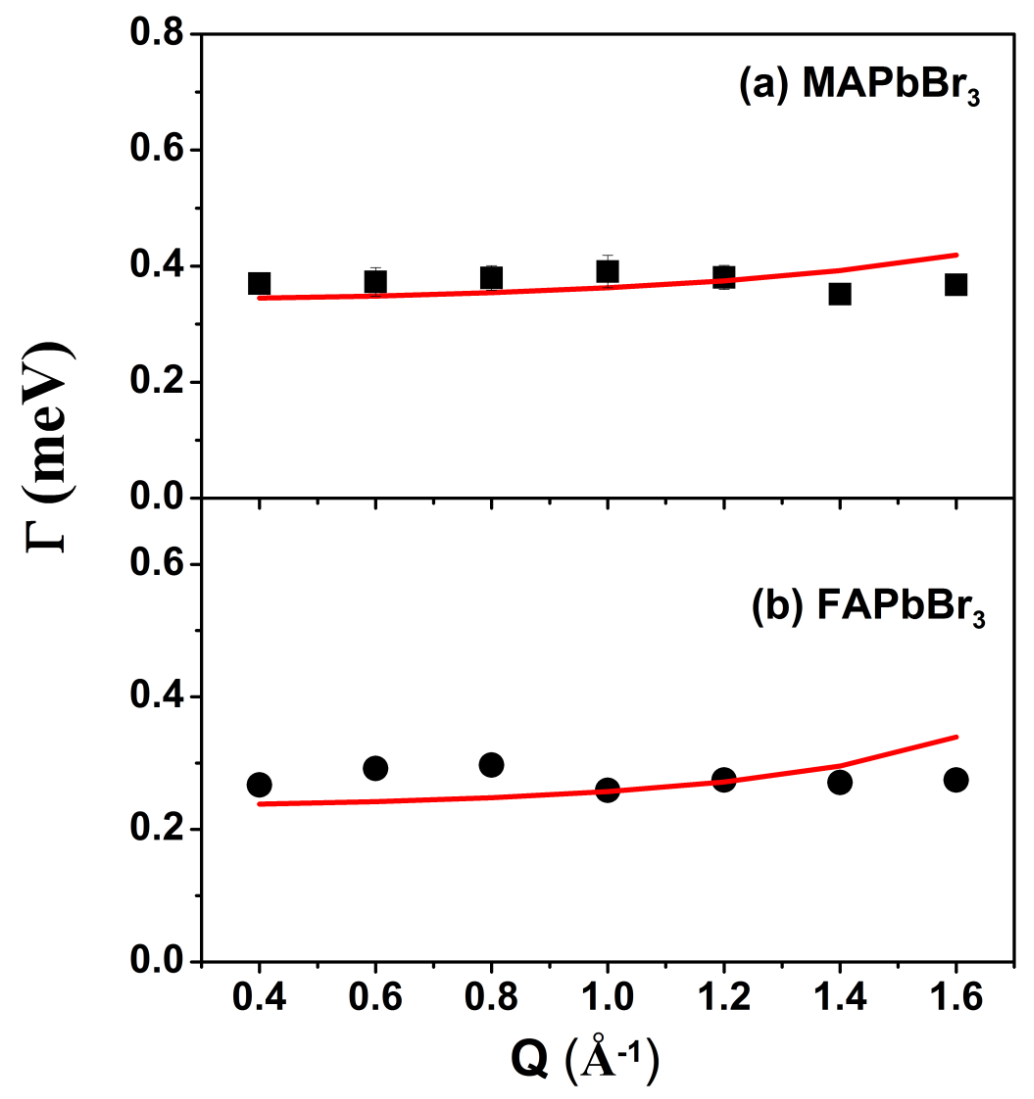

Figure S3. Variation of half width at half maximum of the Lorentzian function for (a) MAPbBr3 and (b) FAPbBr3 at a representative temperature $300 \mathrm{~K}$ with $Q$. Solid lines are the fits according to isotropic rotational diffusion model as described in the text.

\section{References:}

1. Meyer, A.; Dimeo, R. M.; Gehring, P. M.; Neumann, D. A. The high-flux backscattering spectrometer at the NIST Center for Neutron Research. Rev. Sci. Instrum. 2003, 74 (5), 2759-2777.

2. Janßen, S.; Mesot, J.; Holitzner, L.; Furrer, A.; Hempelmann, R. FOCUS: a hybrid TOF-spectrometer at SINQ. Phys. B: Cond. Matt. 1997, 234-236, 1174-1176.

3. Azuah, R. T.; Kneller, L. R.; Qiu, Y.; Tregenna-Piggott, P. L.; Brown, C. M.; Copley, J. R.; Dimeo, R. M. DAVE: A Comprehensive Software Suite for the Reduction, Visualization, and Analysis of Low Energy Neutron Spectroscopic Data. J. Res. Natl. Inst. Stand. Technol. 2009, 114 (1044-677X (Print)), 341.

4. Sears, V. F. Theory of cold neutron scattering by homonuclear diatomic liquids: I. free rotation. Can. J. Phys. 1966, 44 (6), 1279-1297. 